\title{
Effect of melatonin and time of administration on irradiation-induced damage to rat testes
}

\author{
G. Take11, D. Erdogan1, F. Helvacioglu1, G. Göktas¹, G. Ozbey², C. Uluoglu², B. Yücel², \\ Y. Guney ${ }^{4}$, A. Hicsonmez ${ }^{4}$ and S. Ozkan ${ }^{3}$ \\ ${ }^{1}$ Department of Histology and Embryology, ${ }^{2}$ Department of Pharmacology, ${ }^{3}$ Department of Public Healthy, \\ Medical School, Gazi University, Ankara, Turkey \\ ${ }^{4}$ Department of Radiation Oncology, Medical School, Ankara University, Ankara, Turkey
}

Correspondence to: G. Take, Department of Histology and Embriology, Medical School, Gazi University, 06500 Besevler, Ankara, Turkey

Fax: +90-312-212-4647. E-mail: gtake@gazi.edu.tr

\begin{abstract}
The effect of ionizing irradiation on testes and the protective effects of melatonin were investigated by immunohistochemical and electron microscopic methods. Eighty-two adult male Wistar rats were divided into 10 groups. The rats in the irradiated groups were exposed to a sublethal irradiation dose of $8 \mathrm{~Gy}$, either to the total body or abdominopelvic region using a ${ }^{60} \mathrm{Co}$ source at a focus of $80 \mathrm{~cm}$ away from the skin in the morning or evening together with vehicle $(20 \%$ ethanol) or melatonin administered 24 $\mathrm{h}$ before $(10 \mathrm{mg} / \mathrm{kg})$, immediately before $(20 \mathrm{mg} / \mathrm{kg})$ and $24 \mathrm{~h}$ after irradiation $(10 \mathrm{mg} / \mathrm{kg})$, all ip. Caspace-3 immunoreactivity was increased in the irradiated group compared to control $(P<0.05)$. Melatonin-treated groups showed less apoptosis as indicated by a considerable decrease in caspace-3 immunoreactivity $(P<0.05)$. Electron microscopic examination showed that all spermatogenic cells, especially primary spermatocytes, displayed prominent degeneration in the groups submitted to total body and abdominopelvic irradiation. However, melatonin administration considerably inhibited these degenerative changes, especially in rats who received abdominopelvic irradiation. Total body and abdominopelvic irradiation induced identical apoptosis and testicular damage. Chronobiological assessment revealed that biologic rhythm does not alter the inductive effect of irradiation. These data indicate that melatonin protects against total body and abdominopelvic irradiation. Melatonin was more effective in the evening abdominopelvic irradiation and melatonin-treated group than in the total body irradiation and melatonintreated group.
\end{abstract}

Key words: Testes; Gamma-irradiation; Melatonin; Immunohistochemistry; Electron microscopy

Presented at the 4th APICA (Asian-Pacific International Congress of Anatomists), 2005.

The present address of Y. Guney is Department of Radiation Oncology, Ankara Hospital, Ankara, Turkey

Received September 9, 2008. Accepted March 27, 2009

\section{Introduction}

Spermatogenesis is a long, complex and finely tuned process. During this process, the developing sperm cell is sensitive to endogenous or exogenous stresses. Exposure to reproductive cytotoxic agents may damage somatic testicular cells or germ cells at different stages of differentiation, leading to a temporary or permanent impairment of fertility (1). Among reproductive toxic agents, ionizing irradiation has been studied extensively, and is well known to affect testicular function, morphology and spermatogenesis $(2,3)$. Irradiation of the testes can produce reversible or permanent sterility in males (1).

DNA damage induced by ionizing radiation consists mainly of single- and double-strand breaks and, to some extent, base damage. DNA damage can lead to cell death directly or through apoptosis, to the induction of mutations, and to the inhibition of DNA synthesis (4). 
Melatonin ( $\mathrm{N}$-acetyl-5-methoxytryptamine; MW = 232) is an endogenous compound secreted by the pineal gland. It is generally accepted that melatonin influences reproduction via its action on the hypothalamus and the hypothesis has been raised that melatonin may modulate the secretion of gonadotropins $(5,6)$. Once synthesized in the pineal gland, melatonin is quickly released into the bloodstream and then into other body fluids, such as bile, cerebrospinal fluid, saliva, ovarian follicular fluid, semen, and amniotic fluid. Small amounts of non-metabolized melatonin are excreted into urine as well (7). Melatonin concentrations in the body are typically lower during the day and reach maximal levels at night in the dark (8).

Several studies have established that melatonin is a highly efficient free radical scavenger and general antioxidant. Melatonin was also found to scavenge the peroxyl radical, which is generated during peroxidation (9). This implied that melatonin, which is lipophilic and hydrophilic, has effects at the cell level but also within subcellular compartments $(4,10-13)$.

In the present study, we investigated the effects of ionizing radiation applied either to the total body (TB) or only to the abdominopelvic (AP) region on the testes at two different times of day and the protective chronobiological effects of melatonin by immunohistochemical methods using anti-caspace-3, a marker for apoptosis, and electron microscopy to determine irradiation-induced testicular damage.

Table 1. Treatment design for the various groups.

\begin{tabular}{lccccc}
\hline Group & $\begin{array}{c}\text { Time of } \\
\text { irradiation }\end{array}$ & $\begin{array}{c}\text { TB } \\
\text { irradiation }\end{array}$ & $\begin{array}{c}\text { AP } \\
\text { irradiation }\end{array}$ & Vehicle & $\begin{array}{c}\text { Melatonin } \\
\text { treatment }\end{array}$ \\
\hline 1A & Morning & - & - & - & - \\
1B & Evening & - & - & - & - \\
$2 \mathrm{~A}$ & Morning & + & - & + & - \\
2B & Evening & + & - & + & - \\
3A & Morning & - & + & + & - \\
$3 \mathrm{~B}$ & Evening & - & + & + & - \\
$4 \mathrm{~A}$ & Morning & + & - & - & + \\
$4 \mathrm{~B}$ & Evening & + & - & - & + \\
$5 \mathrm{~A}$ & Morning & - & + & - & + \\
$5 \mathrm{~B}$ & Evening & - & + & - & + \\
\hline
\end{tabular}

The experimental groups ( $\mathrm{N}=8$ for each group) were as follows: group 1: control; group 2: total body (TB) irradiation and vehicle (20\% ethanol); group 3: abdominopelvic (AP) irradiation and vehicle; group 4: melatonin with TB irradiation; group 5: melatonin with AP irradiation. The rats in the irradiated groups were exposed to a sublethal irradiation dose of $8 \mathrm{~Gy}$, either to the TB or AP region after ketamine anesthesia using a ${ }^{60} \mathrm{Co}$ source at a focus of $80 \mathrm{~cm}$ from the skin. Administration was in the morning (A) or in the evening (B).

\section{Material and Methods}

\section{Experimental protocol}

Animals. The experimental protocol was approved by the local Ethics Committee for animal studies and conducted at Ankara University Faculty of Medicine. The experiments were performed on male Wistar rats weighing 250-300 g. The animals were fed a standard rat chow diet, had access to water ad libitum and were housed under controlled environmental conditions (light, temperature, feeding time, etc.). The animals were synchronized to a light-dark cycle (lights on from 8:00 am to 8:00 pm) starting at least 2 weeks before the beginning of the experiments. Eighty-two rats were randomly divided into 10 groups ( $N=$ 8 in each group). The treatment design of the groups is shown in Table 1. All experiments were performed during February-March to avoid the possibility of seasonal rhythms affecting the findings (Table 1).

lonizing irradiation. The rats in the irradiated groups were exposed to 8 Gy applied to the TB or AP field following ketamine anesthesia $(100 \mathrm{mg} / \mathrm{kg})$ using a ${ }^{60} \mathrm{Co}$ source at two different times. Morning irradiation was performed $1 \mathrm{~h}$ following lights on (at 9:00 am) and evening irradiation was performed $13 \mathrm{~h}$ following lights on (at 9:00 $\mathrm{pm})$.

Melatonin treatment. Melatonin or vehicle $(20 \%$ ethanol) was administered immediately before, immediately after and $24 \mathrm{~h}$ preceding TB or AP irradiation (melatonin dose: 10,20 , and $10 \mathrm{mg} / \mathrm{kg}$, ip, respectively). Forty-eight hours after irradiation, all animals were sacrificed with ketamine and tissues were obtained.

Immunohistochemical procedure. Testis tissues from each group were fixed in neutral formalin for $72 \mathrm{~h}$ and processed for paraffin embedding. Sections of $4-5-\mu m$ thickness were processed for polylysine-covered microscope slides.

For immunohistochemical examination, slides were stored in a microwave oven in $10 \mathrm{mM}$ Tris- $\mathrm{HCl}$ buffer, (LabVision, USA). Endogenous peroxidase activity was blocked with $3 \%$ hydrogen peroxide (LabVision). Epitopes were stabilized by application of serum blocking solution (LabVision) and slides were then incubated with caspase3 (rabbit polyclonal antibody Ab-4, $1 \mathrm{mg} / \mathrm{mL}$, NewMarker, USA) for $60 \mathrm{~min}$ at room temperature. Next, the biotinylated secondary antibody (goat antirabbit, LabVision), was applied and streptavidin peroxidase (LabVision) was applied to the slides. 3-Amino-9-ethylcarbazole (LabVision) was used as chromogen. Finally, the slides were counterstained with hematoxylin and examined with a light microscope. A negative control was prepared during the stage of primary antibody application. Two independent observers 
who were blind to the treatment regimen evaluated the immunolabeling scores separately.

\section{Electron microscopic procedure}

Tissues were fixed in phosphate buffer containing $2.5 \%$ glutaraldehyde (Sigma-Aldrich, USA) for 2-3 h, post-fixed in $1 \%$ osmium tetraoxide (Sigma-Aldrich) and dehydrated in a series of graded alcohols $(50,60,70,80,90,96$ and $100 \%$ ethanol). After passing through propylene oxide (Sigma-Aldrich), the specimens were embedded in Araldite CY 212 (Ciba-Geigy, USA), (2-dodecen-1-yl)succinic anhydride (Sigma-Aldrich), benzyldimethyl amine (PolySciences Inc., USA) and dibutylphtalate (Sigma-Aldrich). The semi-thin sections were stained with toluidine blue (Sigma-Aldrich), and examined with a photomicroscope (Leica DM4000, Germany). After selection of appropriate specimens, thin sections were cut and stained with uranyl acetate (ProSciTech, Australia) and lead citrate (SigmaAldrich) and examined with an electron microscope (Carl Zeiss EM 900, Germany).

\section{Statistical analysis}

Labeling intensity was graded semiquantitatively and the HSCORE was calculated using the following equation: HSCORE $=P i(i+1)$, where $i$ is the intensity of labeling with a value of 1,2 , or 3 (weak, moderate or strong, respectively) and $\mathrm{P} i$ is the percentage of labeled primary spermatocytes for each intensity, ranging from 0 to $100 \%$. Statistical differences were calculated using the Mann-Whitney U-test. Data are reported as means \pm SEM. A P value $<0.05$ was considered to be significant.

\section{Results}

Immunohistochemical results

Immunohistochemical examination of the testes of the control group (1A) revealed normal seminiferous tubule and interstitial tissue structures. No caspase-3 immunoreactivity was observed in some seminiferous tubules, while a weak immunoreactivity in the spermatogonia and primary spermatocytes was found in other seminiferous tubules in this group. Likewise, no immunoreactivity was observed in the spermatids. The pattern of immunoreactivity was exactly the same in the slides of morning and evening (1B) control groups (data not shown).
In the TB-irradiated group (2A), while the integrity of interstitial tissue was conserved, the organized structure of cells forming the seminiferous tubules was completely disrupted and diffuse intercellular edema was observed in the morning group. In this group, spermatogenesis was absent in most seminiferous tubules. Caspase-3 immunoreactivity was increased from weak to moderate in the spermatogonia, and from moderate to strong in primary spermatocytes. Weak immunoreactivity was observed in the spermatids (Figure 1). Moreover, some degenerative indications were found in the evening group slides (2B). Immunohistochemical evaluation revealed that caspase-3 immunoreactivity was significantly stronger than in the morning group specimens and weak to moderate immunoreactivity was observed in spermatogonia. A strong reactivity was observed in primary spermatocytes although a weak reactivity was observed in the spermatids (data not shown).

In the tissue specimens from animals submitted to TB irradiation in the morning and to melatonin treatment (4A), the testis structure was preserved, and the interstitial tissue within the seminiferous tubules displayed a normal structure. High-power-field examination revealed weak to moderate caspase-3 immunoreactivity in the spermatogonia, diffuse strong reactivity in the primary spermatocytes, and negative to weak reactivity in the spermatids (data not shown). In tissue specimens from the melatonin-treated

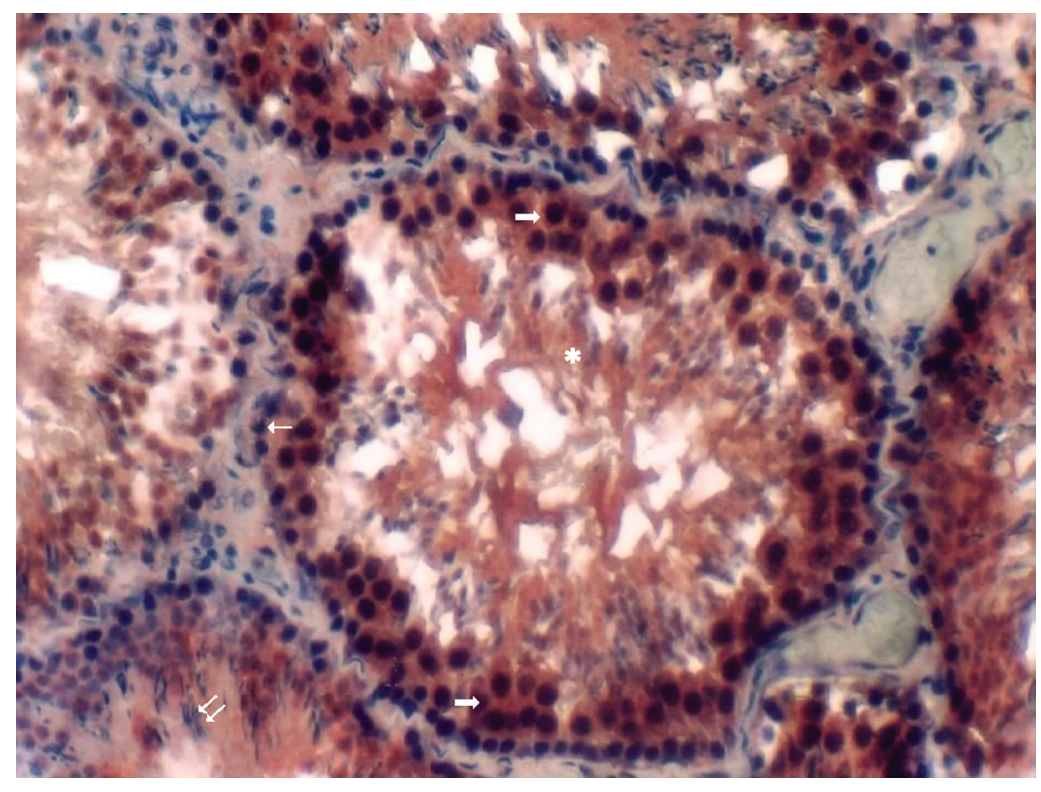

Figure 1. Total body irradiation group (morning). Asterisk: Degenerative seminiferous tubules; thin horizontal arrow: weak-to-moderate immunoreactivity in the spermatogonia; thick horizontal arrows: primary spermatocytes displaying moderate-tostrong immunoreactivity; double oblique arrows: weak spermatid immunoreactivity (immunoperoxidase and hematoxylin staining, 200X). 
evening group (4B), the tissue structure was similar to that observed in the morning group. Caspase- 3 immunoreactivity was observed in a few spermatogonia and primary spermatocytes but was absent throughout the seminiferous tubules. At high magnification, immunoreactivity was weak in the spermatogonia, moderate in the primary spermatocytes and negative in spermatids. Strongly immunoreactive apoptotic bodies were observed within the lumen of the tubules (data not shown).

In AP-irradiated and -untreated tissue specimens of the morning groups $(3 \mathrm{~A})$, the interstitial tissue was preserved, while the seminiferous tubules displayed a normal structure in some fields and interstitial edema in some others. However, the edema was less intense than that observed in the group receiving TB radiation. Spermatogenesis was not detected in these tubules. While caspase3 immunoreactivity was very weak within some tubules, local immunoreactivity was found in some other fields and apoptotic bodies with strong immunoreactivity were noted within the lumen. At high magnification, weak caspase-3 immunoreactivity was observed in the spermatogonia and spermatids, and variable immunoreactivity ranging from moderate to strong was observed within the primary spermatocytes (data not shown). In tissue specimens from animals irradiated in the AP region in the evening (3B), the general structure was similar to that observed in the morn-

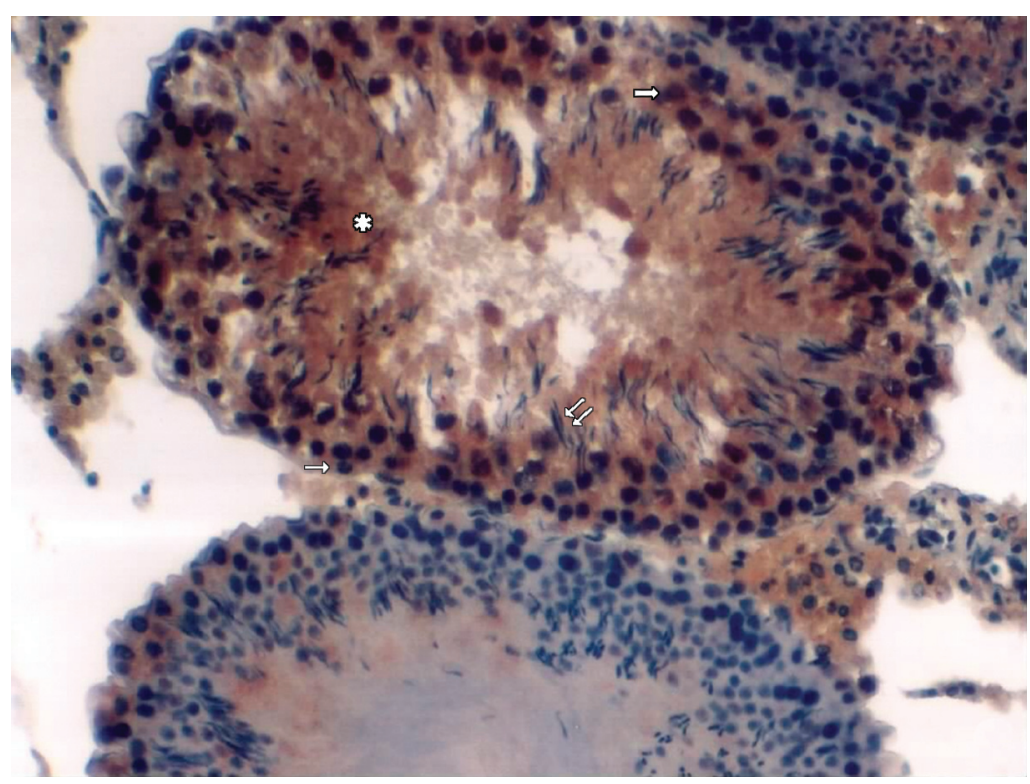

Figure 2. Abdominopelvic irradiation + melatonin (evening). Asterisk: Minimal degenerative changes in seminiferous tubules; thin horizontal arrow: negative-toweak caspase- 3 immunoreactivity in the spermatogonia; thick horizontal arrow: weak-to-moderate immunoreactivity in the primary spermatocytes; double oblique arrows: no immunoreactivity in spermatids (immunoperoxidase and hematoxylin staining, 200X). ing group (3A). Caspase-3 immunoreactivity was similar to that observed in the morning specimens but stronger immunoreactivity was detected in primary spermatocytes (data not shown).

In AP-irradiated and melatonin-treated morning tissue specimens $(5 A)$, prominent edema was observed both in the seminiferous tubules and the interstitial field. Caspase3 immunoreactivity was prominent in some tubules but not in others. Spermatogonia and primary spermatocytes displayed negative immunoreactivity. No immunoreactivity was present in the spermatids (data not shown). Although the AP-irradiated and melatonin-treated evening group (5B) showed more preserved structures than the morning group, caspase-3 immunoreactivity was prominent in some tubules but not in others. In the high-power-field examinations, very weak immunoreactivity was found in the spermatogonia and primary spermatocytes, and negative reactivity in the spermatids. These findings were similar to those observed in controls (Figure 2).

\section{Electron microscopy results}

In the morning and evening control groups, the spermatogenic cells that form seminiferous cords showed a normal ultrastructural appearance. The basement membranes of the seminiferous tubules were also normal (data not shown). In the TB-irradiated morning and evening groups, irregular basement membranes and disconnected junctional complexes were observed between the spermatogenic cells. Some primary spermatocytes looked like apoptotic bodies. A few acrosomal cap formations were determined in these groups because of existing slower spermatogenesis (Figure 3, Panels A and $B)$. In the TB-irradiated and melatonintreated morning and evening groups, the appearance of the spermatogenic cell lines was almost normal, with primary spermatocytes identical to those of the control group. Abundant acrosomal cap formation was noted (data not shown).

On the other hand, different electron microscopy results were observed in the AP-irradiated morning and evening groups. In the morning groups $(3 \mathrm{~A})$, all spermatogenic cell lines were separate from one another and their cytoplasm was filled with diffuse vacuoles (data not shown). In the tissue specimens obtained from the evening groups $(3 \mathrm{~B})$, the general structure was found to be similar to that observed in 


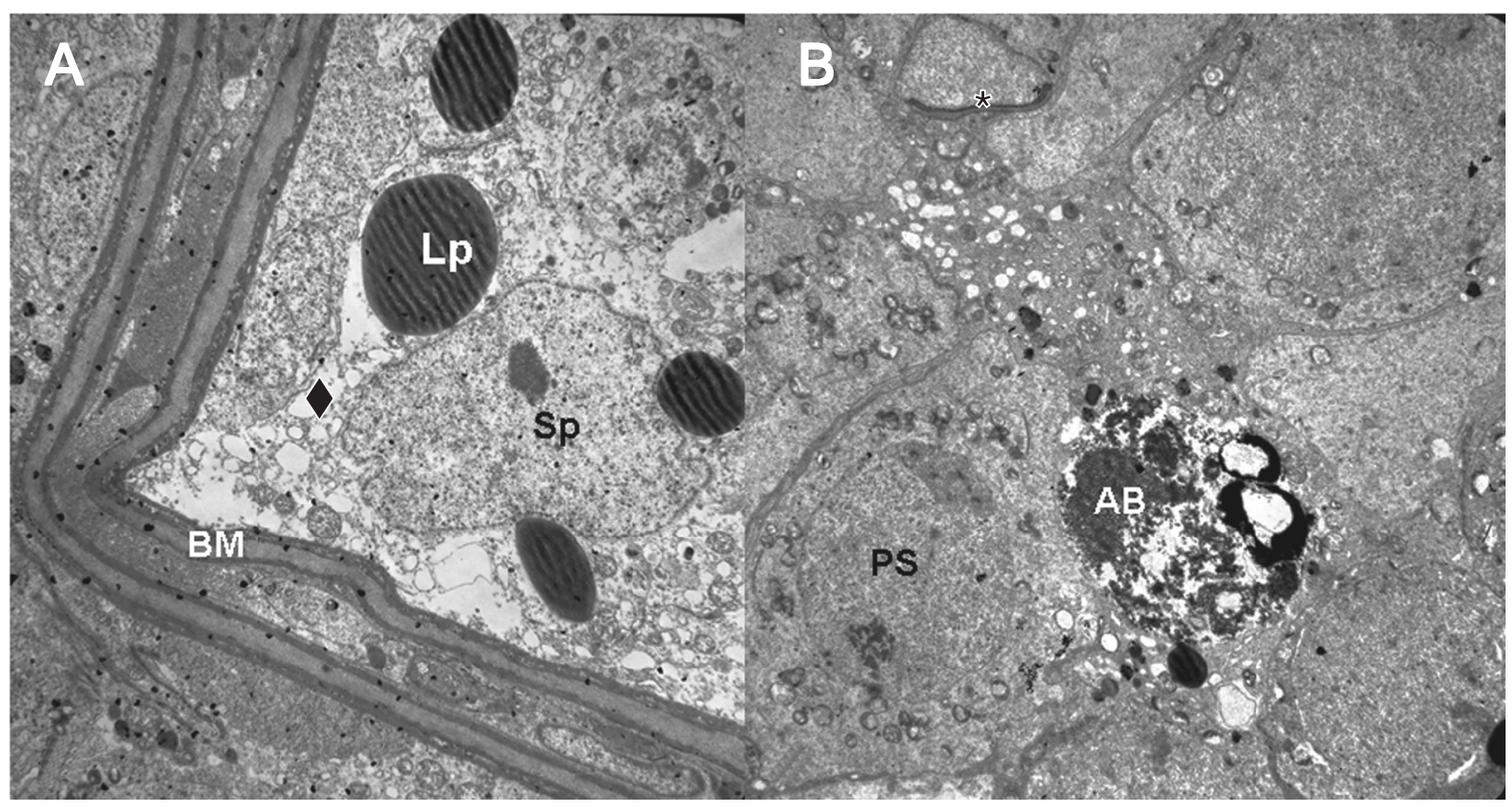

Figure 3. Electron micrograph of the group submitted to total body irradiation. Panel $A$, Spermatogonium (Sp), lipid droplets (Lp), irregular basement membrane (BM), vacuolar formation of disconnected junctional complexes (black lozenge). Panel $B$, Primary spermatocytes (PS), a few acrosomal caps (asterisk) and apoptotic bodies (AB) (uranyl acetate and lead citrate staining, 3000X).

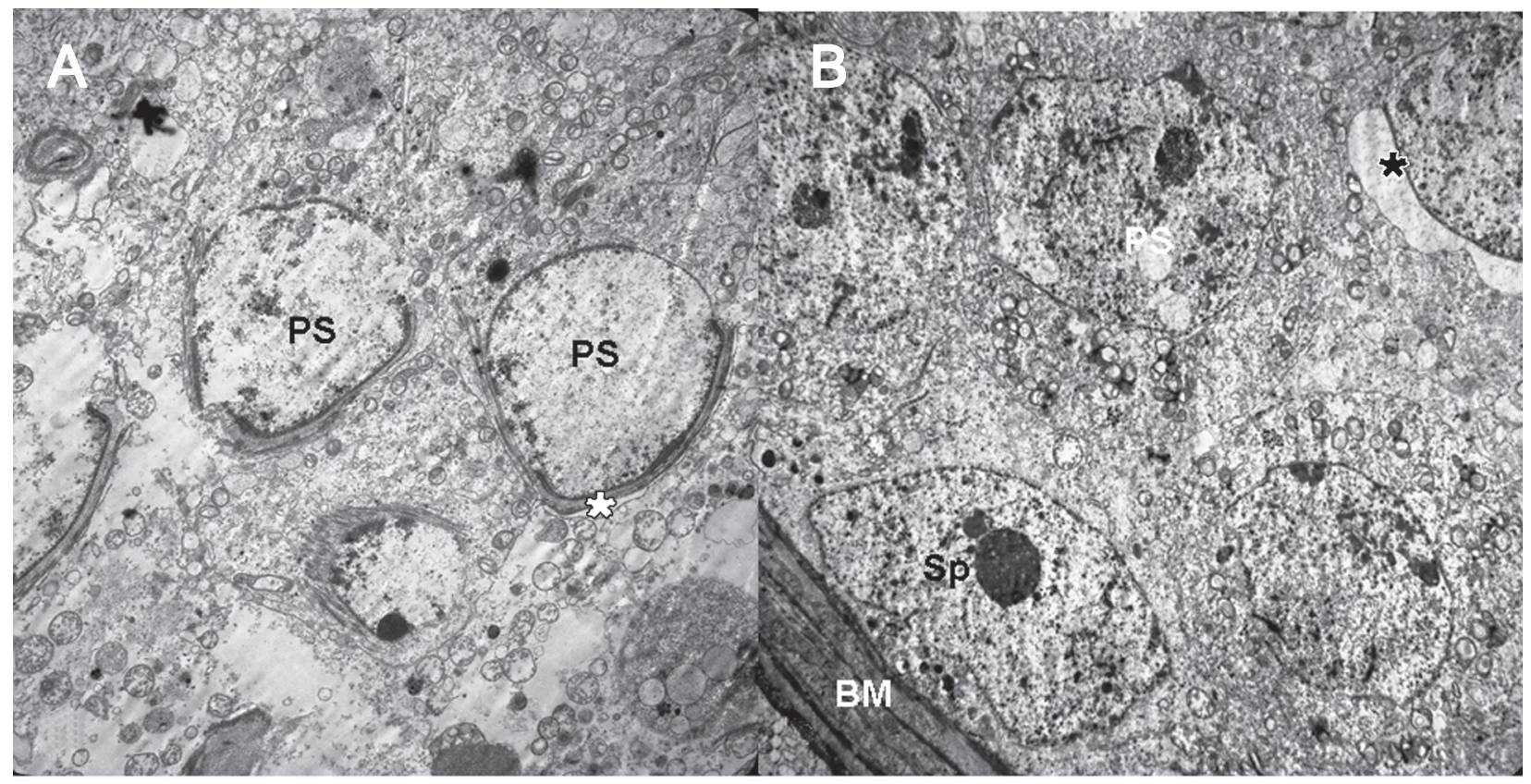

Figure 4. Electron micrograph of the group submitted to abdominopelvic irradiation + melatonin administration. Panel $A$, In tissue samples obtained in the morning, primary spermatocytes show normal structure (PS), with some disconnecting areas between them (asterisk). Panel B, Tissue samples obtained in the evening are similar to control samples, with spermatogonia (Sp), primary spermatocytes (PS), acrosomal caps (asterisk), and basement membrane (BM) (uranyl acetate and lead citrate staining, 3000X). 
Table 2. Immunolabeling intensities of caspase-3 primary spermatocytes from the testes of all experimental groups.

\begin{tabular}{|c|c|c|c|c|c|c|c|c|c|c|}
\hline \multirow[t]{2}{*}{ Reaction } & \multicolumn{2}{|c|}{ Control } & \multicolumn{2}{|c|}{ Total body } & \multicolumn{2}{|c|}{ Total body-melatonin } & \multicolumn{2}{|c|}{ Abdominopelvic } & \multicolumn{2}{|c|}{ Abdominopelvic-melatonin } \\
\hline & Morning & Evening & Morning & Evening & Morning & Evening & Morning & Evening & Morning & Evening \\
\hline Weak & $20.4 \pm 1.5$ & $14.3 \pm 0.1$ & $5.6 \pm 0.8^{a}$ & $3.0 \pm 1^{a b *}$ & $8.6 \pm 0.5^{a c^{*}}$ & $4.2 \pm 0.7^{\mathrm{ad} *}$ & $23.4 \pm 1.1^{\mathrm{ab}}$ & $27.8 \pm 1.0$ & $20.0 \pm 2.3^{c}$ & $16.4 \pm 1.1^{d}$ \\
\hline Moderate & $43.9 \pm 2.9$ & $30.2 \pm 2.0$ & $74.7 \pm 12.5^{*}$ & $26.2 \pm 3.9^{*}$ & $128.5 \pm 2.34^{c^{*}}$ & $73.61 \pm 0.6^{d *}$ & $45.7 \pm 1.9$ & $43.0 \pm 2.1$ & $56.2 \pm 4.0^{\mathrm{C}}$ & $45.3 \pm 1.4^{d}$ \\
\hline Strong & $72.0 \pm 2.7^{*}$ & $32.0 \pm 3.06^{*}$ & $466.3 \pm 19.02^{*}$ & $548.1 \pm 8.5^{\mathrm{b} *}$ & $324.0 \pm 3.06^{c^{*}}$ & $436.7 \pm 11.7^{d}$ & $240 \pm 6.9$ bde & $97.7 \pm 2.9^{d *}$ & $103.1 \pm 6.9$ ce* & $41.03 \pm 5.6^{d *}$ \\
\hline
\end{tabular}

HSCORE values are reported as means \pm SEM. aP $<0.05$ control $v s$ all experimental groups; ${ }^{*} \mathrm{P}<0.05$ morning vs all evening groups; bP $<0.05$ total body morning vs all abdominopelvic morning groups; ${ }^{\mathrm{P}}<0.05$ total body melatonin morning vs all abdominopelvic melatonin morning groups; ${ }^{\mathrm{d}} \mathrm{P}<0.05$ total body melatonin evening $v s$ all abdominopelvic melatonin evening group; eP $<0.05$ abdominopelvic morning $v s$ all abdominopelvic melatonin morning group (Mann-Whitney U-test).

the specimens taken in the morning, but wrinkling of basement membranes was observed (data not shown). In the AP-irradiated and melatonin-treated morning group (5A), separate junctional complexes were observed between the primary spermatocytes, but the remaining tissue seemed to be normal. A normal acrosomal cap formation was detected (Figure 4, Panel A). Finally, in the APirradiated and melatonin-treated evening group (5B) the spermatogenic cell line and tissue in general were similar to control (Figure 4, Panel B).

\section{Statistical results}

Compared to control, the number of immunoreactive primary spermatocytes was markedly increased in all experimental groups, with significant differences between TB and control testes $(P<0.05)$. The number of apoptotic figures was decreased in the primary spermatocytes of the melatonin-treated TB group compared to cells from untreated animals. The differences between the values of TB testes and AP testes were statistically significant $(P<$ 0.05). Similarly, significant differences were observed between TB morning and AP morning testes in terms of the reduction of apoptotic figures $(P<0.05)$. When the TB morning and melatonin-treated TB morning groups were compared to the TB evening and melatonin-treated TB evening groups, the number of apoptotic primary spermatocytes was found to be significantly increased in the evening groups $(P<0.05)$. But we noticed increased apoptotic figures in the AP morning and melatonin-treated AP morning group compared to the same group treated in the evening $(P<0.05)$. The statistical results are shown in Table 2.

\section{Discussion}

Radiation effects are particularly apparent in a rapidly proliferating tissue. The male germ cells in the seminifer- ous epithelium of mammals are among the major cell systems at risk when animals are exposed to irradiation and provide an excellent model for studies in this area (1). Clear degeneration was caused by irradiation of testicular tissue in the present study.

Several researchers have reported the presence of the radiation-mediated degeneration especially in spermatogonia (1,3). Sapp et al. (3) determined that $72 \mathrm{~h}$ after radiation at slightly higher doses, the spermatogonial response begins to differ with type of irradiation. When exposed to Xrays, about $30 \%$ of the total spermatogonial population is lost, with 0.10-Gy exposure inducing a sharp decrease in the surviving fraction. However, with exposures from 0.1 to 0.8 Gy the decrease in survival fraction is much more gradual. Approximately an additional $40 \%$ of the spermatogonial population is lost within this range of exposures. Thirty percent of the total population remain intact, even at 0.8 Gy. However, between 0.8 and 1 Gy there is another sharp decline in cells. In the present study, in contrast to the literature, apoptosis was lower in the spermatogonia of samples taken from groups submitted to morning and evening TB and AP irradiation, a result probably due to the short period of irradiation.

Different opinions exist in the literature about the protective effects of melatonin against irradiation treatment $(4,11)$. Badr et al. (4) suggested that the radioprotective effect of melatonin is not species-specific and acts in a similar way in different biological systems. Administration of melatonin after animal exposure to radiation had no significant effect. However, the effect of melatonin per se doubled the frequency of micronuclei in bone marrow polyerythrocytes compared to control. This suggests a cytotoxic effect of melatonin at the relatively high dose of $10 \mathrm{mg} / \mathrm{kg}$ body weight. The cytotoxic effect of exogenous aspirated melatonin noted in this study may be due to its abnormal concentration in the circulation.

In the present study, melatonin was found to have 
protective effects and to be more effective in the evening groups and in the endogenous form, a fact probably related to the dose applied. Thus, in clinical therapy, the dose applied is thought to be a very important point in the progression of treatment.

Mornjakovic et al. (14) determined the volume density of the seminiferous epithelium, tubular lumen and testis interstitium in sham-pinealectomized adult Wistar rats after melatonin treatment and whole-body irradiation with 8 Gy gamma rays. They found that melatonin is able not only to modify the quantitative characteristics of the seminiferous tubules but also to reduce the effects originally produced by irradiation. Also, melatonin administration can significantly reduce the notorious effects of irradiation on Leydig cells (15). Taken together, several observations support the notion that melatonin has a radioprotective effect against $\mathrm{X}$-ray irradiation. First, melatonin administration prior to irradiation prevented radiation damage to peripheral blood cells (16). Second, 6- and 8-Gy X-ray irradiation of rats was associated with increased malondialdehyde, myeloperoxidase and nitric oxide levels and decreased glutathione levels (17). All of these indices were reduced with melatonin pretreatment (18). Therefore, melatonin, by its free radical scavenging and antioxidant properties, ameliorates irradiation-induced cell damage (17).

In the present study, we investigated the protective effect of melatonin after both TB and AP applications. In TB-irradiated and morning melatonin-treated testis specimens (4A), the tissue structure was occasionally preserved, whereas caspase- 3 immunoreactivity was similar to that of the group undergoing radiation exposure without melatonin treatment. The 4B group showed results similar to control. In the group receiving both AP irradiation and melatonin treatment, no reaction was observed in the majority of the tubules in the tissue specimens taken in the morning. In light of these findings, we propose that AP irradiation has weaker effects on the spermatogonia than TB irradiation, and therefore the radioprotective effects of melatonin administration/treatment were more prominent in the group that underwent abdominal radiation.

In addition, our findings differed considerably from those in the literature, particularly in that we found prominent effects of radiation on primary spermatocytes, which were considerably improved following melatonin administration. We also found that the cells most sensitive to the difference between the morning and evening periods were primary spermatocytes. We attributed this sensitivity to the fact that the primary spermatocytes were in the phase of proliferation, and therefore displayed denser caspase-3 reactivity compared with the other cell groups when exposed to environmental factors such as irradiation.
The Sertoli and Leydig cells of seminiferous tubule and their basement membranes have been investigated in ultrastructural studies (19-21), but there are no studies in the literature dealing with the protective effects of melatonin on the ultrastructure of radiated testes.

Hussein et al. (22) reported ultrastructural features of apoptosis (condensation of the nuclei, vacuolization of the cytoplasm, increased cytoplasmic density, and apoptotic bodies) in irradiated testes, which were absent when the irradiated animals were pretreated with melatonin. There was minimal depletion of Sertoli and Leydig cells following $\mathrm{X}$-ray irradiation. Also, morphological features of apoptosis were infrequent in these cells. The authors found that the protective effects included amelioration of germ-cell depletion and apoptotic changes.

In contrast to the above studies, in the present study AP irradiation was also applied in addition to TB irradiation and spermatocytes were assayed. The groups submitted to TB irradiation in the morning and evening presented irregular basement membranes and disconnected spermatogenic cell line junctional complexes, features that were not observed in TB-irradiated + melatonin-treated morning and evening groups. Furthermore, in AP-irradiated morning groups (3A), all spermatogenic cell lines were separate from one another and their cytoplasm was filled with diffuse vacuoles. In the AP-irradiated evening groups (3B), the general structure was found to be similar to that observed in the AP-irradiated morning groups, but wrinkling of the basement membranes was observed. Also, in the AP-irradiated and melatonin-treated morning group $(5 A)$, separated junctional complexes were observed between the primary spermatocytes, but the remainder of the tissue appeared to be normal. Finally, in the AP-irradiated and melatonin-treated evening group (5B), the spermatogenic cell line and tissue in general were similar to control.

To the best of our knowledge, this study is the first to report the immunohistochemical (caspase-3, both quantitative and qualitative analyses) and ultrastructural changes following administration of melatonin in TB- and AP-irradiated testes. Our data suggest a radioprotective role for melatonin mostly against testis damage induced by AP evening irradiation. We discovered that radiation therapy causes most prominent degeneration and increased apoptosis in the primary spermatocytes of the spermatogenetic cord. The presence of increased caspace- 3 reactivity and apoptosis following TB and AP irradiation supports the view of the detrimental effects of these rays. The mechanisms underlying the present observations, as well as their possible clinical and therapeutic ramifications, require further investigations. 


\section{References}

1. Cordelli E, Fresegna AM, Leter G, Eleuteri $P$, Spano $M$, Villani $P$. Evaluation of DNA damage in different stages of mouse spermatogenesis after testicular $\mathrm{X}$ irradiation. Radiat Res 2003; 160: 443-451.

2. Sapp WJ, Philpott DE, Williams CS, Williams JW, Kato K, Miquel JM, et al. Comparative study of spermatogonial survival after X-ray exposure, high LET (HZE) irradiation or spaceflight. Adv Space Res 1992; 12: 179-189.

3. Sapp WJ, Philpott DE, Williams CS, Kato K, Stevenson J, Vasquez M, et al. Effects of spaceflight on the spermatogonial population of rat seminiferous epithelium. FASEB $J$ 1990; 4: 101-104.

4. Badr FM, El Habit OH, Harraz MM. Radioprotective effect of melatonin assessed by measuring chromosomal damage in mitotic and meiotic cells. Mutat Res 1999; 444: 367-372.

5. Kim JK, Lee CJ. Effect of exogenous melatonin on the ovarian follicles in gamma-irradiated mouse. Mutat Res 2000; 449: 33-39.

6. Vera $\mathrm{H}$, Tijmes M, Ronco AM, Valladares LE. Melatonin binding sites in interstitial cells from immature rat testes. Biol Res 1993; 26: 337-340.

7. Vijayalaxmi, Reiter RJ, Tan DX, Herman TS, Thomas CR Jr. Melatonin as a radioprotective agent: a review. Int J Radiat Oncol Biol Phys 2004; 59: 639-653.

8. Claustrat B, Geoffriau M, Brun J, Chazot G. [Melatonin in humans: a biochemical marker of the circadian clock and an endogenous synchronizer]. Neurophysiol Clin 1995; 25: 351-359.

9. lanas O, Olinescu R, Badescu I. Melatonin involvement in oxidative processes. Endocrinologie 1991; 29: 147-153.

10. Pieri $C$, Marra M, Moroni $F$, Recchioni $R$, Marcheselli $F$. Melatonin: a peroxyl radical scavenger more effective than vitamin E. Life Sci 1994; 55: L271-L276.

11. Reiter RJ. Interactions of the pineal hormone melatonin with oxygen-centered free radicals: a brief review. Braz $\mathrm{J}$ Med Biol Res 1993; 26: 1141-1155.

12. Reiter RJ. Functional pleiotropy of the neurohormone melatonin: antioxidant protection and neuroendocrine regulation.
Front Neuroendocrinol 1995; 16: 383-415.

13. Reiter R, Tang L, Garcia JJ, Munoz-Hoyos A. Pharmacological actions of melatonin in oxygen radical pathophysiology. Life Sci 1997; 60: 2255-2271.

14. Mornjakovic Z, Scepovic M, Kundurovic Z. [Morphometric aspects of seminiferous tubules in rats treated with melatonin and whole body irradiation (stereologic analysis)]. Med Arh 1991; 45: 9-10.

15. Mornjakovic Z, Alicelebic S, Bilalovic N, Susko I. [Morphometric characteristics of Leydig cells after total irradiation of rats treated with melatonin]. Med Arh 1998; 52: 183-184.

16. Koc M, Buyukokuroglu ME, Taysi S. The effect of melatonin on peripheral blood cells during total body irradiation in rats. Biol Pharm Bull 2002; 25: 656-657.

17. Sener G, Jahovic N, Tosun O, Atasoy BM, Yegen BC. Melatonin ameliorates ionizing radiation-induced oxidative organ damage in rats. Life Sci 2003; 74: 563-572.

18. Taysi S, Koc M, Buyukokuroglu ME, Altinkaynak K, Sahin YN. Melatonin reduces lipid peroxidation and nitric oxide during irradiation-induced oxidative injury in the rat liver. $J$ Pineal Res 2003; 34: 173-177.

19. Pinon-Lataillade $G$, Velez de la Calle JF, Viguier-Martinez MC, Garnier DH, Folliot R, Maas J, et al. Influence of germ cells upon Sertoli cells during continuous low-dose rate gamma-irradiation of adult rats. Mol Cell Endocrinol 1988; 58: 51-63.

20. Dedov VI. [Ultrastructure of rat Sertoli and Leydig cells normally and under prolonged internal irradiation]. Tsitologiia 1980; 22: 1153-1156.

21. Sawada H, Esaki M. Electron microscopic observation of 137Cs-irradiated rat testis: production of basal laminae for germ cells, despite their absence. J Electron Microsc 2003; 52: 391-397.

22. Hussein MR, Abu-Dief EE, Abou El-Ghait AT, Adly MA, Abdelraheem $\mathrm{MH}$. Morphological evaluation of the radioprotective effects of melatonin against $X$-ray-induced early and acute testis damage in Albino rats: an animal model. Int $J$ Exp Pathol 2006; 87: 237-250. 